\title{
Palmitoylethanolamide counteracts hepatic metabolic inflexibility modulating mitochondrial function and efficiency in diet-induced obese mice
}

\author{
Chiara Annunziata $^{1}$ | Adriano Lama ${ }^{1}$ | Claudio Pirozzi ${ }^{1}$ | Gina Cavaliere ${ }^{2}$ | \\ Giovanna Trinchese $^{2}$ | Francesca Di Guida ${ }^{1}$ | Allegra Nitrato Izzo ${ }^{1}$ | Fabiano Cimmino ${ }^{2}$ \\ Orlando Paciello $^{3}$ | Davide De Biase ${ }^{3}$ | Elisabetta Murru ${ }^{4}$ | Sebastiano Banni ${ }^{4}$ | \\ Antonio Calignano $^{1}$ | Maria Pina Mollica ${ }^{2} \mid$ Giuseppina Mattace Raso $^{1} \mid$ Rosaria Meli $^{1}$
}

${ }^{1}$ Department of Pharmacy, University of
Naples Federico II, Naples, Italy
${ }^{2}$ Department of Biology, University of
Naples Federico II, Naples, Italy
${ }^{3}$ Department of Veterinary Medicine and
Animal Production, University of Naples
Federico II, Naples, Italy
${ }^{4}$ Department of Biomedical Sciences,
University of Cagliari, Cittadella
Universitaria di Monserrato, Cagliari, Italy

\section{Correspondence}

Maria Pina Mollica, Department of Biology, University of Naples Federico

II, Complesso Universitario di Monte

Sant'Angelo, Cupa Nuova Cinthia

21-Edificio 7, 80126 Naples, Italy.

Email: mariapia.mollica@unina.it

\begin{abstract}
Peroxisome proliferator-activated receptor (PPAR)- $\alpha$ activation controls hepatic lipid homeostasis, stimulating fatty acid oxidation, and adapting the metabolic response to lipid overload and storage. Here, we investigate the effect of palmitoylethanolamide (PEA), an endogenous PPAR- $\alpha$ ligand, in counteracting hepatic metabolic inflexibility and mitochondrial dysfunction induced by high-fat diet (HFD) in mice. Long-term PEA administration (30 mg/kg/die per os) in HFD mice limited hepatic lipid accumulation, increased energy expenditure, and markedly reduced insulin resistance. In isolated liver mitochondria, we have demonstrated PEA capability to modulate mitochondrial oxidative capacity and energy efficiency, leading to the reduction of intracellular lipid accumulation and oxidative stress. Moreover, we have evaluated the effect of PEA on mitochondrial bioenergetics of palmitate-challenged HepG2 cells, using Seahorse analyzer. In vitro data showed that PEA recovered mitochondrial dysfunction and reduced lipid accumulation in insulin-resistant HepG2 cells, increasing fatty acid oxidation. Mechanistic studies showed that PEA effect on lipid metabolism was limited by AMP-activated protein kinase (AMPK) inhibition, providing evidence for a pivotal role of AMPK in PEA-induced adaptive metabolic setting. All these findings identify PEA as a modulator of hepatic lipid and glucose homeostasis, limiting metabolic inflexibility induced by nutrient overload.
\end{abstract}

\footnotetext{
Abbreviations: ACC, acetyl-CoA carboxylase; AKT, protein kinase B; AMPK, adenosine monophosphate kinase; ALT, alanine aminotransferase; AST, aspartate aminotransferase; AUC, area under curve; CPT, carnitine-palmitoyltransferase; CSV, Institutional Animal Care and Use Committee; FAO, fatty acid oxidation; FCCP, p-trifluoromethoxy phenylhydrazone; GAPDH, glyceraldehyde-3-phosphate dehydrogenase; GSH, reduced glutathione; GSSG, oxidized glutathione; H\&E, hematoxylin \& eosin; HFD, high-fat diet; HOMA, Homeostasis Model Assessment; IL, interleukin; IR, insulin resistance; InsR, insulin receptor; ITT, insulin tolerance test; MCP1, monocyte chemoattractant protein-1; NAEs, N-acylethanolamines; NAFLD, non-alcoholic fatty liver disease; OCR, oxygen consumption rate; OGTT, oral glucose tolerance test; Pal, sodium palmitate; PEA, palmitoylethanolamide; PPARs, peroxisome proliferator-activated receptors; PTT, pyruvate tolerance test; ROS, reactive oxygen species; RQ, respiratory quotient; SOD, superoxide dismutase; STD, standard diet; TNF, tumor necrosis factor.
}

Chiara Annunziata, Adriano Lama, and Claudio Pirozzi contributed equally to the study as first authors.

Giuseppina Mattace Raso and Rosaria Meli contributed equally to the study as senior authors. 


\section{$1 \mid$ INTRODUCTION}

Metabolic flexibility is the ability of several tissues (ie, liver, adipose tissue, skeletal muscle, and heart) to adapt metabolism and manage nutrient sensing, uptake, transport, storage, and expenditure, switching from fatty acid oxidation (FAO) during fasting to glucose metabolism during the fed state. Impaired metabolic flexibility has been reported to be associated with obesity and type 2 diabetes, but it is still unknown whether this detrimental condition is a cause or a consequence of these pathologies. ${ }^{1}$ Upon consumption of a high-fat diet (HFD), lean individuals with adequate metabolic flexibility are able to induce FAO compared to obese ones, increasing the expression of genes involved in fatty acid transport and oxidation. ${ }^{2}$ The inflexibility, implicated in the ectopic accumulation of lipid and in the development of insulin resistance (IR), ${ }^{3}$ is improved by weight loss. ${ }^{4}$ The pathogenesis of IR is based on an imbalance between energy consumption and expenditure that leads to lipid accumulation. IR is crucial for the pathogenesis of type 2 diabetes; therefore, the improvement of insulin signaling pathway leads to the restoration of energy homeostasis and insulin sensitivity, as an effective strategy for the treatment of type 2 diabetes.

In the liver, impaired FAO produces toxic lipid intermediates altering insulin signaling pathway and triggering oxidative stress and subsequent mitochondrial dysfunction. ${ }^{5,6}$ Recently, mitochondria emerged as key regulators of metabolic flexibility selecting fuel in response to nutritional changes and substrate disposal. ${ }^{6,7}$

In non-alcoholic fatty liver disease (NAFLD), chronic toxic lipid overload causes the activation of inflammatory pathways, resulting in increased pro-inflammatory adipokines and reduced insulin-sensitizing adipokines. ${ }^{8}$ Besides collecting nutrients, portal blood drains different mediators released from gut and visceral adipose tissue. ${ }^{9}$ Therefore, hepatic metabolism is directly affected not only by nutrients assumed from the diet but also by the secretory profile of visceral fat, highlighting the endocrine and immune interactions in NAFLD-related diseases.

Peroxisome proliferator-activated receptors (PPARs) are lipid sensors that modulate metabolic pathways in response to substrate disposal. Indeed, PPAR- $\alpha$, as a ligand-activated transcription factor, exerts positive and/or negative control over the expression of a range of metabolic and inflammatory genes ${ }^{10}$ and regulates cognitive flexibility in mice. ${ }^{11}$ The ability of this nuclear receptor to integrate metabolic and inflammatory pathways makes it an attractive target for intervention in metabolic diseases, such as NAFLD and IR. ${ }^{12-14}$

Palmitoylethanolamide (PEA, C16:0), an endogenous PPAR- $\alpha$ agonist, has shown to have a bi-faced pharmacological profile. Indeed, beyond the well-known analgesic and anti-inflammatory effects, ${ }^{15}$ PEA shows a metabolic activity, modulating energy balance in animals ${ }^{16}$ and humans. ${ }^{17}$

Here, we have investigated PEA metabolic activities in liver, focusing on glucose and lipid homeostasis in a mouse model of diet-induced obesity. Moreover, our mechanistic study provides evidence for a central role of AMP kinase (AMPK) in PEA-induced adaptive metabolic setting.

\section{MATERIALS AND METHODS}

\subsection{Ethics statement}

All procedures involving the animals were carried out in accordance with the international and national law and policies (EU Directive 2010/63/EU for animal experiments, ARRIVE guidelines and the Basel declaration, including the "3R" concept). All animal procedures reported herein were approved by the Institutional Animal Care and Use Committee (CSV) of the University of Naples Federico II under protocol no. 982/2017-PR.

\subsection{Animals and in vivo experimental procedures}

Male C57B1/6J mice (Harlan, Italy) at 6 weeks of ages were housed in stainless steel cages in a room kept at $22 \pm 1^{\circ} \mathrm{C}$ with a 12:12 hours light-dark cycle. Standard chow diet (Mucedola srl, Milan, Italy) had $17 \%$ fat, without sucrose while HFD (Research Diets Inc, NJ, USA) had $45 \%$ of energy derived from fat, $7 \%$ of sucrose. Standard and HFD contained 15.8 and $21.9 \mathrm{~kJ} / \mathrm{g}$, respectively, determined by bomb calorimeter. Moreover, the diet composition formula, the detailed fatty acid profile, and the relative percentage of monounsaturated and saturated fatty acid of STD diet and HFD are reported in Table S1. The total energy intake was calculated considering the weekly food intake and the energy content of the diets.

After weaning, young mice were randomly divided into three groups ( $\mathrm{n}=30$ animals) as follows: (i) control group 
(STD) receiving chow diet and vehicle per os by gavage; (ii) HFD group receiving vehicle; (iii) HFD group treated with PEA (HFD+PEA, $30 \mathrm{mg} / \mathrm{kg} /$ die per os, by gavage). The treatments started after 12 weeks of HFD feeding and lasted 8 weeks. Ultra-micronized PEA was provided by Epitech Group Research Labs (Padova, Italy). It was suspended in carboxymethyl cellulose (1.5\%) for oral gavage. During the experimental period, body weight was weekly assessed. At the end of the experimental protocol, before sacrifice, bioelectrical impedance analysis was performed to determine fat body composition assessment using BIA 101 analyzer, modified for the mouse (Akern, Florence, Italy). The animals were anaesthetized by enflurane followed by cervical dislocation and serum and tissues were collected. Liver from all mice was removed and the samples not immediately used for mitochondria preparation were frozen and stored at $-80^{\circ} \mathrm{C}$ for subsequent biochemical determinations.

\subsection{Measurement of oxygen consumption, carbon dioxide production, and respiratory quotient}

Following an adaption period to the experimental environment, oxygen consumption $\left(\mathrm{VO}_{2}\right)$ and carbon dioxide production $\left(\mathrm{VCO}_{2}\right)$ were recorded by a monitoring system (Panlab s.r.l., Cornella, Barcelona, Spain) that is composed of a four-chambered indirect open-circuit calorimeter, designed for continuous and simultaneous monitoring. $\mathrm{VO}_{2}$ and $\mathrm{VCO}_{2}$ were measured every 15 minutes (for 3 minutes) in each chamber for a total of 6 hours. The mean $\mathrm{VO}_{2}, \mathrm{VCO}_{2}$, respiratory quotient $(\mathrm{RQ})$ values, and energy expenditure were calculated by the "Metabolism H" software. ${ }^{18}$

\subsection{Glucose, insulin, and pyruvate tolerance tests}

At the beginning of $8^{\text {th }}$ week of PEA treatment, oral glucose tolerance test (OGTT), insulin tolerance test, (ITT) and pyruvate tolerance test (PTT) were performed on different subgroups of mice (10 animals/each group).

OGTT was performed in overnight fasted animals receiving glucose (1 g/kg, Sigma-Aldrich, St Louis, MO, USA), whereas insulin $(0.75 \mathrm{IU} / \mathrm{kg}$, Humulin, Lilly, Indianapolis, IN, USA) was intraperitoneally injected in fed mice to assess ITT. Blood glucose levels were measured at selected time points after injection. The area under the curve (AUC) was calculated from time 0 , as an integrated and cumulative measure of glycemia up to 120 minutes for all animals. For PTT, fasted animals were i.p. injected with pyruvate $(2 \mathrm{~g} /$ $\mathrm{kg}$, Sigma-Aldrich, St Louis, MO, USA) and glycemia was measured at 15, 30, 60, and 120 minutes after the administration. Glucose levels were measured by the glucometer One Touch Ultrasmart (Lifescan, Milpitas, CA).

\subsection{Serum and hepatic parameters and tissue isolation}

At the end of the experimental period ( $8^{\text {th }}$ week), the sera and livers were collected. In serum, alanine aminotransferase (ALT), aspartate aminotransferase (AST), triglycerides, and total cholesterol were measured by colorimetric enzymatic method using commercial kits (SGM Italia, Italy and Randox Laboratories ltd., United Kingdom). Serum interleukin (IL)-1, IL-10 (Thermo Scientific, Rockford, IL, USA), tumor necrosis factor (TNF)- $\alpha$ (Biovendor $R \& D$, Brno, Czech Republic), adiponectin and leptin (B-Bridge International Mountain View, CA), and fasting insulin (cat. no. EZRMI-13K; Millipore, Darmstadt, Germany) concentrations were measured using commercially available ELISA kits. As the index of IR, HOmeostasis Model Assessment (HOMA)-IR was calculated, using the formula $[$ HOMA $=$ fasting glucose $(\mathrm{mmol} / \mathrm{L}) \times$ fasting insulin $(\mu \mathrm{U} / \mathrm{mL}) / 22.5]$. Liver samples were homogenized in saline solution and then centrifuged at $5000 \mathrm{rpm}$ for 5 minutes. Supernatants were collected and centrifugated at $14000 \mathrm{rpm}$ at $4^{\circ} \mathrm{C}$ for 15 minutes and triglycerides quantified (TGL Flex reagent cartridge, Siemens Healthcare $\mathrm{GmbH}$, Erlangen, Germany). Liver tissues, obtained from all animal groups, were excised for further biochemical and histological analysis.

\section{6 | Hepatic histological and fatty acids analysis}

Livers were collected and either preserved in $10 \%$ neutral buffered formalin or snap frozen in Isopentane precooled in liquid nitrogen. Formalin-fixed and paraffin embedded $4 \mu \mathrm{m}$ sections were stained with hematoxylin and eosin (H\&E) for morphology. Cryostat $10 \mu \mathrm{m}$ liver sections were stained with Oil Red O (\# 04-220923, Bio Optica, Milan, Italy) according to the manufacturer's instruction to measure intracellular lipid droplet accumulation. A double-blinded examination of the sections was made by veterinary pathologists at a magnification $\times 200$ with a concordance rate of $95 \%$. Statistical analysis was performed using SPSS software $(P<.05)$. The comparison among groups was performed using ANOVA univariate analysis.

Analysis of fatty acids (palmitic, palmitoleic, and oleic acids) and PEA was carried out as described by Piscitelli et al. ${ }^{19}$ The values ( $\mathrm{n}=6$ each group) are expressed in pmoles or $\mu$ moles/g liver tissue. 


\section{7 | Measurements of mitochondrial oxidative capacity and degree of coupling}

In another set of animals, the mitochondrial isolation, oxygen consumption, and the degree of coupling measurements were performed as previously reported. ${ }^{20}$

The degree of coupling was determined in the liver by applying equation by Cairns et $\mathrm{al}^{21}$ : degree of coupling $=\sqrt{1-(J o)_{s h} /(J o)_{\text {unc }}}$ where $(J o)_{\text {sh }}$ represents the oxygen consumption rate in the presence of oligomycin that inhibits ATP synthase, and $(\mathrm{Jo})_{\text {unc }}$ is the uncoupled rate of oxygen consumption induced by carbonyl cyanide p-trifluoromethoxy phenylhydrazone (FCCP), which dissipates the transmitochondrial proton gradient. $(\mathrm{Jo})_{s h}$ and $(\mathrm{Jo})_{\text {unc }}$ were measured as above using succinate $(10 \mathrm{mmol} / \mathrm{L})$, rotenone $(3.75 \mu \mathrm{mol} / \mathrm{L})$ in the presence of oligomycin $(2 \mu \mathrm{g} / \mathrm{mL})$ or FCCP $(1 \mu \mathrm{mol} / \mathrm{L})$, respectively.

Carnitine-palmitoyltransferase (CPT) total activity was followed spectrophotometrically as $\mathrm{CoA}-\mathrm{sH}$ production by the use of 5,5'-dithiobis (nitrobenzoic acid) (DTNB) and as substrate palmitoyl CoA $10 \mu \mathrm{M}$. The medium consisted of 50 $\mathrm{mM} \mathrm{KCl}, 10 \mathrm{mM}$ Hepes (pH 7.4), 0.025\% Triton X-100, 0.3 $\mathrm{mM}$ DTNB, and 10-100 pg of mitochondrial protein in a final volume of $1.0 \mathrm{~mL}$. The reaction was followed at $412 \mathrm{~nm}$ with spectrophotometer, and enzyme activity was calculated from an $\mathrm{E} 412=13600 /(\mathrm{M} \times \mathrm{cm})$. The temperature was maintained at $25^{\circ} \mathrm{C}^{22,23}$

\section{$2.8 \quad$ Oxidative stress}

The levels of reactive oxygen species (ROS) were also determined in liver homogenate as previously reported..$^{20}$ The specific activity of aconitase and superoxide dismutase (SOD) was spectrophotometrically measured. ${ }^{22,24}$ Catalase activity was determined based on the decomposition of $\mathrm{H}_{2} \mathrm{O}_{2}$ at $25^{\circ} \mathrm{C} .{ }^{25}$ Reduced glutathione (GSH) and oxidized glutathione (GSSG) concentrations in the liver were measured with the dithionitrobenzoic acid-GSSG reductase recycling assay. ${ }^{26}$

\section{9 | Cell culture experiments}

Human HepG2 cells (American Type Culture Collection, Manassas, VA) were cultured in RPMI 1640 complete medium at $37^{\circ} \mathrm{C}$ with $5 \% \mathrm{CO}_{2}$. After 16-hour starvation in 5\% FBS medium, HepG2 cells were incubated with sodium palmitate (Pal, $100 \mu \mathrm{M}$, Sigma-Aldrich, Milan, Italy) or its vehicle, for 24 hours to obtain Pal-induced insulin resistant and control cells, respectively. ${ }^{27,28} 2$ hours before Pal challenge, cells were pre-treated with PEA $(1 \mu \mathrm{M})$. When used, compound C $(2 \mu \mathrm{M})$, an inhibitor of AMPK (Sigma-Aldrich, Milan, Italy), was added 1 hour before PEA stimulation.
After 24-hour Pal challenge, cell lipid content was evaluated $^{29}$ or Western blot analysis on cell lysate was performed.

\subsection{Cellular oxygen consumption measurement}

HepG2 cells were seeded in Seahorse XFp Analyzer (Agilent Technologies, Santa Clara, CA, USA) in mini plates at 30000 cells/well in RPMI growth medium overnight using Mito Stress Test. Cells were treated with the tested compounds as described above; then, the medium was replaced with $750 \mu \mathrm{L}$ unbuffered Seahorse XF Base medium supplemented with glucose $(10 \mathrm{mM})$, L-glutamine $(2 \mathrm{mM})$, sodium pyruvate $(1 \mathrm{mM})$, equilibrated at $37^{\circ} \mathrm{C}$ in a $\mathrm{CO}_{2}$-free incubator for 1 hour, following manufacturer's instructions. Respiration was expressed as oxygen consumption rate (OCR, $\mathrm{pmol} / \mathrm{min}$ ). The proton leak was determined after inhibition of mitochondrial ATP production by $1 \mu \mathrm{M}$ oligomycin (as an inhibitor of the F0/F1 ATPase). Afterward, the mitochondrial electron transport chain was maximally stimulated by the addition of the uncoupler FCCP $(2 \mu \mathrm{M})$. The extra-mitochondrial respiration was estimated after the injection of rotenone $(1 \mu \mathrm{M})$ and antimycin $\mathrm{A}(0.5 \mu \mathrm{M})$, inhibitors of the complexes I and III, respectively. Coupling efficiency is the proportion of the consumed oxygen to drive ATP synthesis compared with that driving proton leak ( $\%$ of ATP-linked OCR/basal OCR). ${ }^{30}$ OCR was normalized to the protein content of each well for all measurements by Bradford assay. ${ }^{31}$

\subsection{Histological score analysis}

For histological examination, three main broad categories of histological features were analyzed: steatosis, inflammation, and necrosis. The grading system was adapted from Kleiner et al. $^{32}$ as previously described. ${ }^{33}$ Kleiner's grading system considers the following histological variables: severity of steatosis (quantified by low- to medium-power evaluation of parenchymal involvement by steatosis): score $0,<5 \%$; score 1 , $5-33 \%$; score 2, $>33-66 \%$, score $3,>66 \%$; location (predominant distribution pattern): zone 3 , score 0 ; zone 1 , score 1 ; azonal, score 2; inflammation: lobular inflammation (overall assessment of all inflammatory foci): score 0 , no foci; score 1 , $<2$ foci per $\times 200$ magnification field; score 2, 2-4 foci per $\times 200$ magnification field; score $3,>4$ foci per $\times 200$ magnification field; necrosis: score 0 , present; score 1 , absent.

\subsection{2 | Western blotting}

Livers, after homogenization, and HepG2 cells were lysed and total protein lysates were undergone to SDS-PAGE. The 
filter was probed with a rabbit polyclonal antibody against anti-GLUT2 (Millipore Corporation, Billerica, MA, USA), anti-phospho protein kinase B (AKT) or anti-AKT, antiphosphoAMPK (pAMPK) or anti-AMPK, anti-phospho acetyl-CoA carboxylase (pACC) (Ser 79) or anti-ACC, anti-insulin receptor (InsR) (Cell Signaling Technology, Danvers, MA, USA), or anti-phospho InsR (pInsR, Thermo Scientific, Rockford, USA), anti-PPAR- $\alpha$ (Sigma-Aldrich, Milan, Italy), anti-PPARGC1- $\alpha$ (PGC1- $\alpha$, Elabscience, Houston, Texas), and anti-CPT1 (Santa Cruz Biotechnology, Inc., Santa Cruz, CA). Western blot for (GAPDH), Tubulin, and $\beta$-Actin (Sigma-Aldrich, Milan, Italy) were performed to ensure equal sample loading. Bands were detected by ChemiDoc imaging instrument (Bio-Rad, Segrate, Italy).

\subsection{Real-time semi-quantitative PCR}

Total RNA, isolated from liver, was extracted following protocols as previously reported. ${ }^{34}$ Each cDNA sample (500 ng) was mixed with 2X QuantiTech SYBRGreen PCR Master Mix and primers, Ppara, Fasn, Tnfa, Il6, and Ucp2 (Qiagen, Hilden, Germany). The relative amount of each studied mRNA was normalized to Actb as a housekeeping gene, and the data were analyzed according to the $2^{-\Delta \Delta C t}$ method. Realtime PCR was performed by CFX96 instrument (Bio-Rad, Segrate, Italy).

\subsection{Statistical analysis}

Data are presented as mean value \pm SEM or SD. Statistical analysis was performed by one- or two-way ANOVA followed by Bonferroni's post-hoc, for multiple comparisons. Differences among groups were considered significant at values of $P<.05$; different superscripted letters indicate significant statistical differences.

Analyses were performed using GraphPad Prism 7 (GraphPad Software, San Diego, CA, USA).

\section{3 | RESULTS}

\section{1 $\quad$ PEA reduced lipid accumulation, increased energy expenditure, and resting metabolic rate}

The mean body weight of all groups was reported, starting 12 weeks after HFD feeding throughout the 8-week treatment period (Figure 1A). PEA treatment gradually reduced body weight, reaching significance after 7 weeks. Consistently, 8-week treatment with PEA reduced fat mass (Figure 1B). Total energy intake of HFD+PEA group was lower than that of HFD (Figure 1C). Moreover, PEA treatment increased energy metabolism, as shown by the higher $\mathrm{VO}_{2}$ consumption and $\mathrm{VCO}_{2}$ production (Figure 1D) compared to HFD mice. Notably, RQ ratio decreased in PEA-treated HFD mice indicating an increase in FAO (Figure 1E). Consistently, the energy expenditure of HFD + PEA mice was increased compared to HFD ones (Figure 1F).

\section{2 | PEA modulated serum parameters, improved glucose metabolism, and hepatic insulin sensitivity}

In Table 1, biochemical and hormonal parameters are reported. Serum triglycerides, cholesterol, ALT, and TNF$\alpha$, increased by HFD, were significantly reduced by PEA. Similarly, the hormonal profile altered by HFD was improved by PEA treatment, as shown by leptin and adiponectin serum levels, whose ratio was consistently reduced. Moreover, PEA treatment reduced serum insulin and glucose levels, resulting in a reduction in the HOMA-IR index.

Glucose metabolism was analyzed (Figure 2); HFD induced a marked increase in glycemia curve in OGTT and AUC value, which were significantly reduced by PEA (Figure 2A). Similarly, peripheral glucose sensitivity was improved following insulin injection in PEA-treated mice, as shown by the increased glucose disposal and AUC (Figure 2B). Moreover, pyruvate administration in PEAtreated animals reduced glucose levels at all examined times compared to HFD mice, indicating the reduction of gluconeogenesis (Figure 2C).

Insulin signaling and GLUT2 expression were reduced in the liver of mice on HFD, revealing IR. PEA treatment increased insulin sensitivity, restoring the phosphorylation of InsR and AKT, and inducing GLUT2 protein expression (Figure 2D-F).

\section{3 | PEA reduced liver damage and improved hepatic lipid metabolic impairment}

The histological pattern of HFD mice was characterized by micro- and macro-vesicular steatosis with prominent ballooning. Lobular inflammation was characterized by inflammatory cells occasionally arranged in microgranulomas (see arrow) and consisting mostly in lymphocytes, plasma cells, and neutrophils (Figure 3A). Liver sections of HFD+PEA animals showed a mild reduction of zone 1 micro-vesicular steatosis and ballooning, accompanied by a reduction of inflammatory lesions and absence of necrosis (Figure 3B). Moreover, PEA reduced hepatic lipid accumulation caused by HFD feeding, as shown by the slight positivity to ORO staining (Figure 3A). Accordingly, the increase in hepatic 


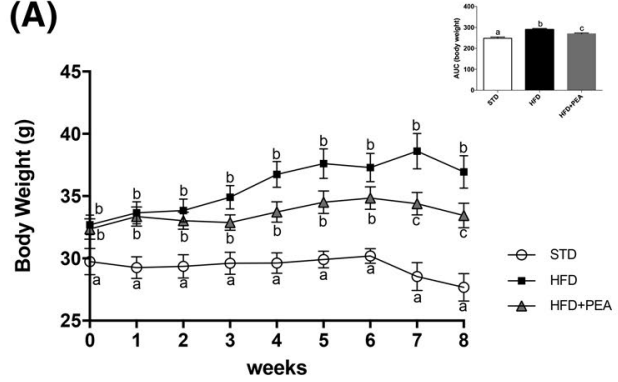

(C)

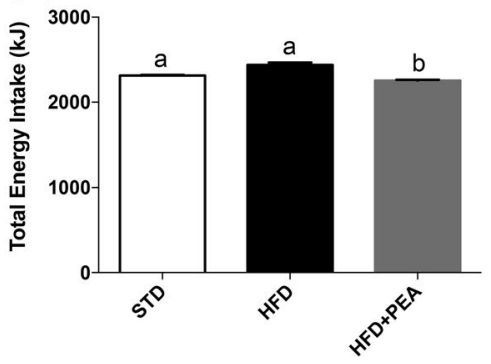

(E)

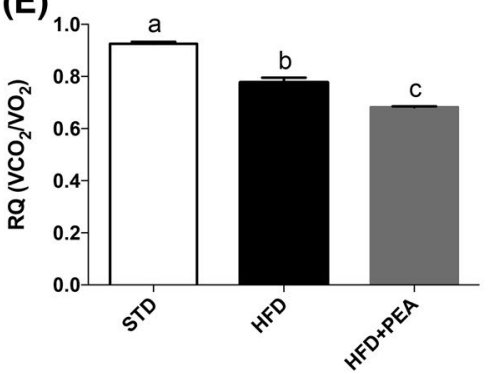

(B)

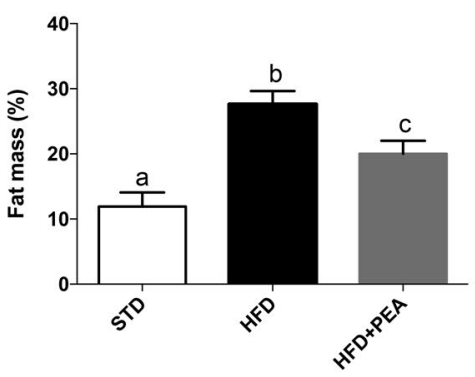

(D)

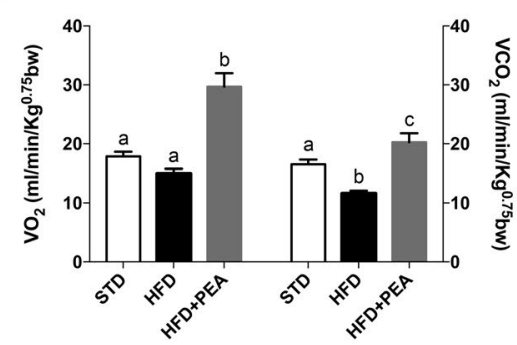

(F)

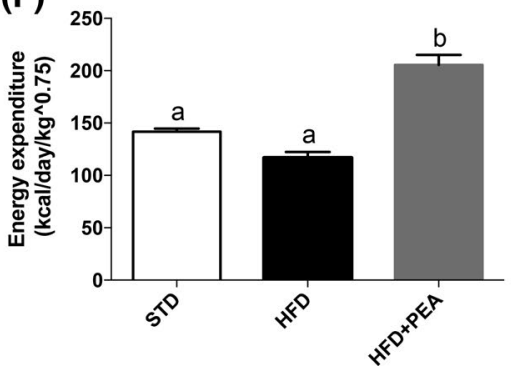

F I G U RE 1 PEA reduced body weight and increased energy expenditure in diet-induced obese mice. A, Effect of PEA treatment on body weight measured throughout the treatment period (0-8 weeks after 12-week HFD feeding), B, Fat mass measured by bioelectrical impedance analysis and $\mathrm{C}$, Total energy intake was obtained considering weekly food intake and dietary gross density energy $\left(\mathrm{n}=10\right.$ each group). $\mathrm{D}, \mathrm{VO}_{2}$ consumption and $\mathrm{VCO}_{2}$ production, $\mathrm{E}, \mathrm{RQ}\left(\mathrm{VCO}_{2} / \mathrm{VO}_{2}\right), \mathrm{F}$, Energy expenditure were determined at the end of experimental period by an opencircuit calorimeter $(\mathrm{n}=5$ each group). Data are presented as means \pm SEM. Labeled means without a common letter differ, $P<.05$

triglycerides of HFD mice was reduced by PEA treatment (Figure 3C). Notably, HFD decreased significantly hepatic PEA levels that were normalized by PEA oral treatment. Palmitic acid, palmitoleic acid, and oleic acids were increased by HFD and unchanged in HFD+PEA treated mice (Table 2).

We also demonstrated that PEA increased Ppara and reduced Fasn transcription (Figure 3D), together with the increased phosphorylation of AMPK and its downstream target ACC (Figure 3E,F), the main pathway involved in FAO. Moreover, we showed that PEA induced the protein expression of hepatic PPAR- $\alpha$ and its co-activator PGC1 $\alpha$ and that of CPT1 (Figure 4A,B).

Based on the extended hepatic inflammation grade of HFD mice, we explored Il6 and Tnfa mRNA expression, two inflammatory mediators implicated in the pathogenesis of steatosis and IR. PEA-treated HFD mice showed a reduction in both cytokine transcription levels, confirming PEA antiinflammatory profile in HFD-induced steatosis (Figure 3G).

\subsection{Modulation of hepatic mitochondrial efficiency and oxidative stress by PEA treatment}

Mitochondrial state 3 respiration, evaluated using succinate as substrate, was decreased in HFD-fed animals compared with the other groups and restored by PEA (Figure 5A). To study FAO, state 3 respiration was evaluated using palmitoylcarnitine as substrate; PEA increased oxygen consumption compared with STD and HFD groups (Figure 5B). No variation was observed in mitochondrial state 4 respiration among all groups using succinate or palmitoyl-carnitine substrate (Figure 5A,B, respectively). High quality of mitochondrial preparations was indicated by high respiratory control ratio values in all groups (data not shown). CPT activity did not differ between STD- and HFD-fed mice while it was increased by PEA treatment (Figure 5C). To test mitochondrial efficiency, we measured oxygen consumption in the 


\begin{tabular}{|lccr|}
\hline & \multicolumn{1}{l}{ STD } & HFD & HFD + PEA \\
\hline Triglycerides $(\mathrm{mg} / \mathrm{dL})$ & $116.1 \pm 5.124^{\mathrm{a}}$ & $213.6 \pm 3.618^{\mathrm{b}}$ & $151.2 \pm 4.505^{\mathrm{c}}$ \\
\hline Total Cholesterol $(\mathrm{mg} / \mathrm{dL})$ & $163.7 \pm 4.479^{\mathrm{a}}$ & $227.6 \pm 4.694^{\mathrm{b}}$ & $190.9 \pm 2.489^{\mathrm{c}}$ \\
\hline Serum ALT $(\mathrm{U} / \mathrm{L})$ & $70.14 \pm 2.064^{\mathrm{a}}$ & $124.1 \pm 3.894^{\mathrm{b}}$ & $98 \pm 4.416^{\mathrm{c}}$ \\
\hline Serum TNF- $\alpha(\mathrm{ng} / \mathrm{mL})$ & $0.9514 \pm 0.0504^{\mathrm{a}}$ & $3.777 \pm 0.1205^{\mathrm{b}}$ & $1.202 \pm 0.2694^{\mathrm{a}}$ \\
\hline Leptinemia $(\mathrm{ng} / \mu \mathrm{L})$ & $1.29 \pm 0.0626^{\mathrm{a}}$ & $18.4 \pm 0.9042^{\mathrm{b}}$ & $14.77 \pm 0.4992^{\mathrm{c}}$ \\
\hline Adiponectinemia $(\mu \mathrm{g} / \mathrm{mL})$ & $3.099 \pm 0.08055^{\mathrm{a}}$ & $1.19 \pm 0.09502^{\mathrm{b}}$ & $2.688 \pm 0.1893^{\mathrm{a}}$ \\
\hline Ratio $($ leptin/adiponectin) & $0.4185 \pm 0.02418^{\mathrm{a}}$ & $16.09 \pm 1.516^{\mathrm{b}}$ & $5.702 \pm 0.6046^{\mathrm{c}}$ \\
\hline Insulin $(\mathrm{ng} / \mathrm{dL})$ & $0.394 \pm 0.01451^{\mathrm{a}}$ & $1.545 \pm 0.5375^{\mathrm{b}}$ & $0.4443 \pm 0.0321^{\mathrm{a}}$ \\
\hline Glucose $(\mathrm{mg} / \mathrm{dL})$ & $93.5 \pm 3.794^{\mathrm{a}}$ & $135.4 \pm 6.871^{\mathrm{b}}$ & $102.5 \pm 90.31^{\mathrm{a}}$ \\
\hline HOMA index & $1.744 \pm 0.04303^{\mathrm{a}}$ & $5.646 \pm 1.467^{\mathrm{b}}$ & $2.783 \pm 0.3655^{\mathrm{c}}$ \\
\hline
\end{tabular}

T A B L E 1 Biochemical and hormonal serum parameters are reported

Note: Data are expressed as mean \pm SEM ( $\mathrm{n}=6$ for each group). Labeled means without a common letter differ, $P<0.05$.

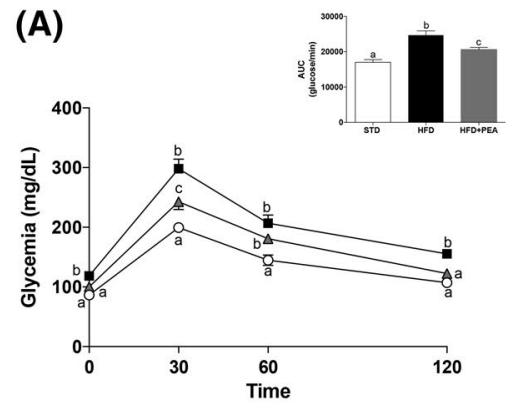

(D)
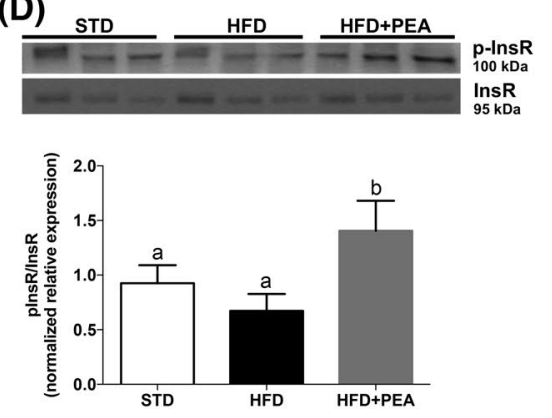

(B)

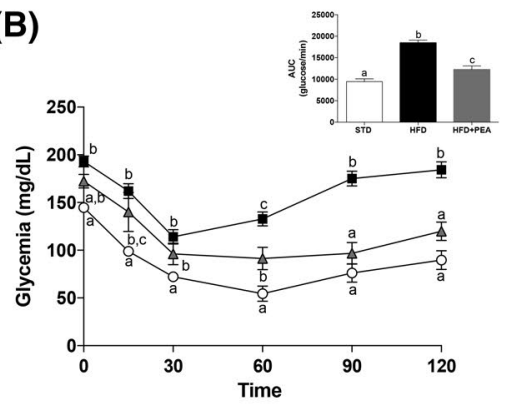

(E)
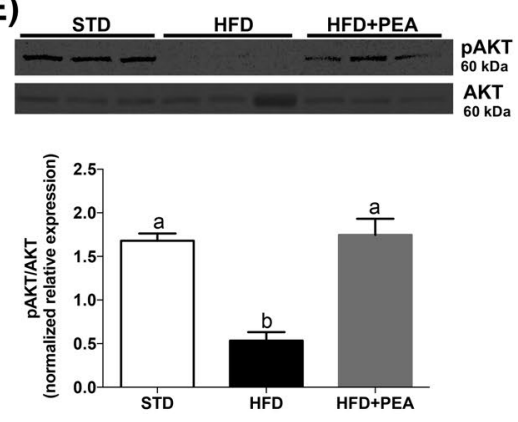

(C)

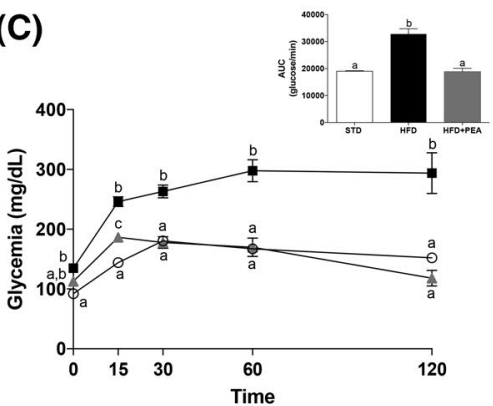

(F)
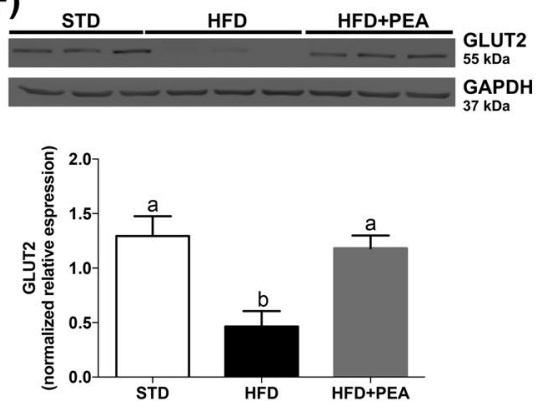

F I G U RE 2 Effect of PEA on glucose homeostasis. A, Oral glucose tolerance test (OGTT), B, Insulin and C, Pyruvate tolerance tests (ITT and PTT, respectively) were performed in all groups of mice. Data are presented as means \pm SEM ( $\mathrm{n}=10$ animals each group). D, Liver pInsR-to-InsR, and E, pAKT-to-AKT ratios and F, GLUT2 expression were also evaluated by Western blot and densitometric analysis reported. Data are presented as means \pm SEM of animals from different groups $(n=4-6$ each group). Labeled means without a common letter differ, $P<.05$

presence of oligomycin and FCCP (Figure 5D). Oligomycin state 4 respiration showed a significant reduction in HFD animals compared to STD, whereas it was significantly increased in PEA-treated mice (Figure 5D). No variation was found in maximal FCCP-stimulated respiration (Figure 5D). Therefore, hepatic mitochondrial energetic efficiency, assessed as the degree of coupling, was increased in HFD and decreased by PEA treatment (Figure 5E). This reduction in energy efficiency is associated to PEA-induced increase in mRNA expression of uncoupling protein (UCP) 2 (Figure $5 \mathrm{~F}) . \mathrm{UCP} 2$ is an anion transporter involved in mitochondrial calcium homeostasis and is related to the increase in proton leak and the reduction of ROS production. ${ }^{35}$

Hepatic ROS production, increased in HFD mice, was significantly decreased by PEA (Figure 6A). Accordingly, PEA increased antioxidant defense, promoting SOD, aconitase, and catalase activity (Figure 6B-D). Moreover, while the hepatic GSH level was increased by PEA treatment, no difference in GSSG content was shown among groups (Figure 6E). The beneficial effects on liver redox status induced by PEA were clearly indicated by the significant increase of the GSH/GSSG ratio (Figure 6F). 

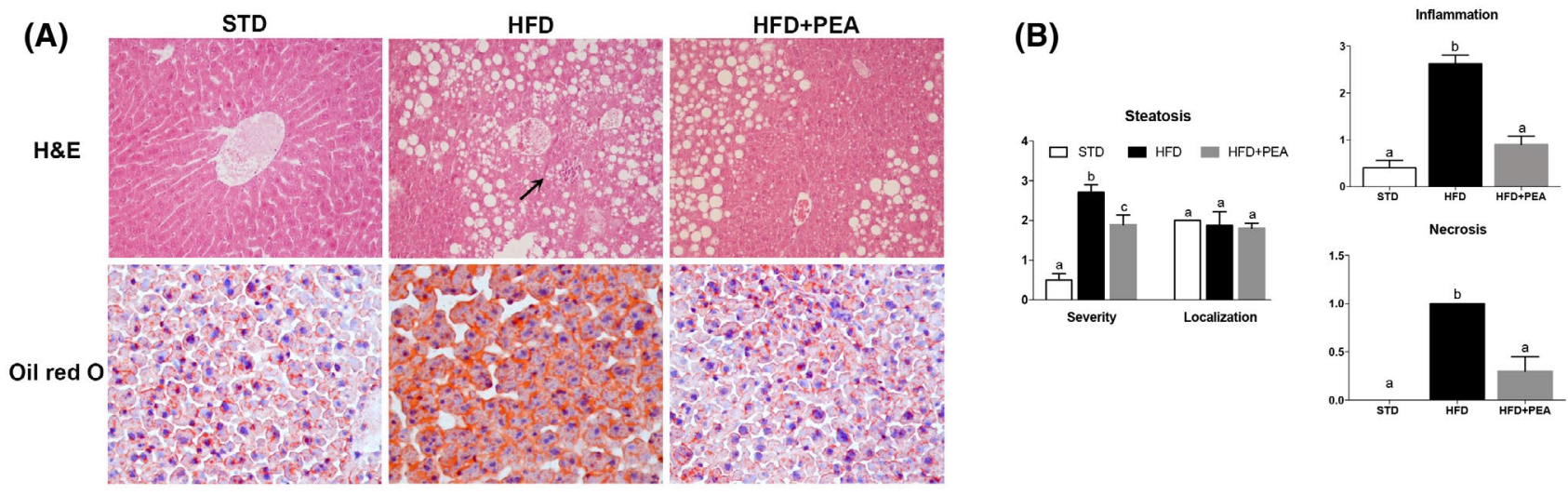

(C)

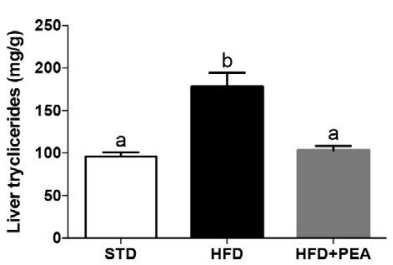

(F)
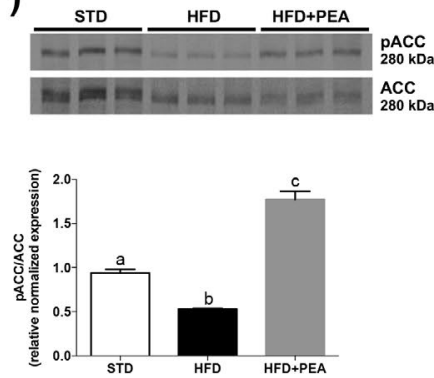

(D)

$\square$ STD $\square$ HFD $\square$ HFD+PEA

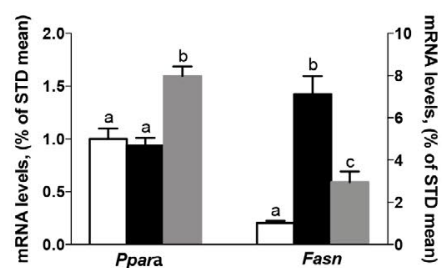

(E)

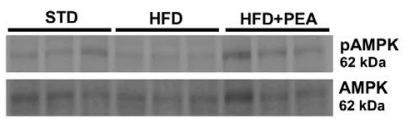

(G)

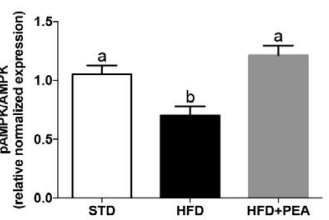

F I G U RE 3 PEA effect on liver lipid accumulation in HFD-fed mice. A, Paraffin-embedded sections of the liver ( $\mathrm{n}=4$ each group) were stained with H\&E and Oil Red O. Micrographs are representative pictures with original magnification $\times 20$. B, Effect of PEA treatment on severity of steatosis, hepatic inflammation, and necrosis was also evaluated by Kleiner's grading system. C, Liver triglycerides content, D, Ppara and Fasn mRNA levels, E, Liver pAMPK-to-AMPK, F, pACC-to-ACC were evaluated by Western blot and densitometric analysis shown. G, Il6 and Tnfa mRNA level are reported. Data are presented as means \pm SEM of animals from different groups ( $n=4-6$ each group). Labeled means without a common letter differ, $P<.05$

TA B L E 2 Determination of PEA and long-chain fatty acids (palmitic, palmitoleic, and oleic acids) in mouse liver

\begin{tabular}{cccc} 
& STD & HFD & HFD + PEA \\
\hline PEA (pmoles/g tissue) & $24.824 \pm 2.873^{\mathrm{a}}$ & $11.432 \pm 0.562^{\mathrm{b}}$ & $22.796 \pm 1.840^{\mathrm{a}}$ \\
\hline $\begin{array}{c}\text { Palmitic Acid (16:0) } \\
(\mu \text { moles/g tissue) }\end{array}$ & $35.228 \pm 1.911^{\mathrm{a}}$ & $44.614 \pm 4.326^{\mathrm{b}}$ & $44.654 \pm 4.448^{\mathrm{b}}$ \\
$\begin{array}{c}\text { Palmitoleic acid }(16: 1) \\
(\mu \text { moles/g tissue) }\end{array}$ & $2.723 \pm 0.496^{\mathrm{a}}$ & $6.042 \pm 0.810^{\mathrm{b}}$ & $4.219 \pm 0.528^{\mathrm{b}}$ \\
$\begin{array}{c}\text { Oleic acid }(18: 1) \\
(\mu \text { moles/g tissue) }\end{array}$ & $12.794 \pm 1.577^{\mathrm{a}}$ & $39.350 \pm 6.395^{\mathrm{b}}$ & $32.423 \pm 3.720^{\mathrm{b}}$ \\
\hline
\end{tabular}

Note: Data are expressed in pmoles or $\mu$ moles/g liver tissue ( $\mathrm{n}=6$ each group), as mean \pm SD. Labeled means without a common letter differ, $P<0.05$. 
(A)

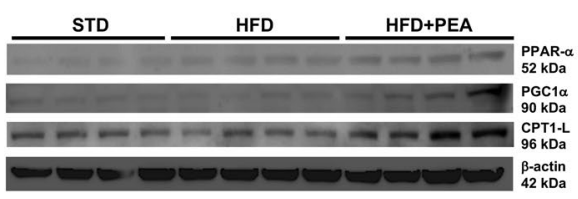

(B)
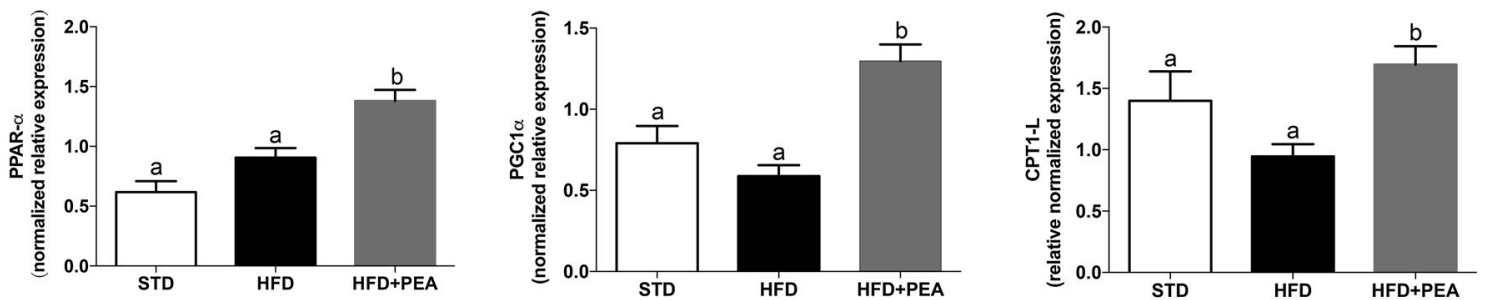

F I G U RE 4 PEA induced PPAR- $\alpha$ and PGC1 $\alpha$ expression in HFD mice. A, In liver, we evaluated protein level of PPAR- $\alpha$, the coactivator PCG1 $\alpha$, and its downstream target CPT1-L and B, Densitometric analysis was reported. Data are presented as means \pm SEM of animals from different groups ( $\mathrm{n}=4-6$ each group). Labeled means without a common letter differ, $P<.05$.

(A)

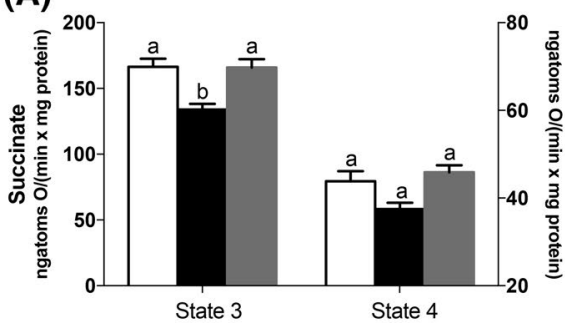

STD

(C)

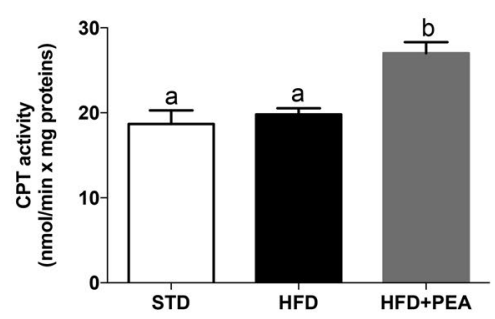

(E)

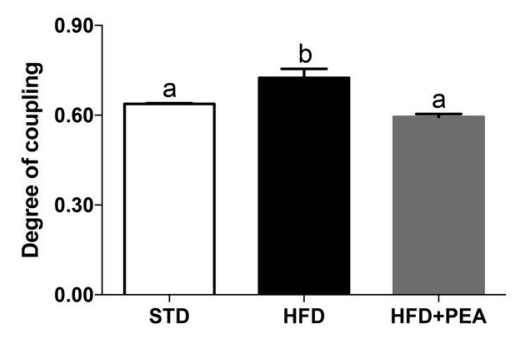

(B)

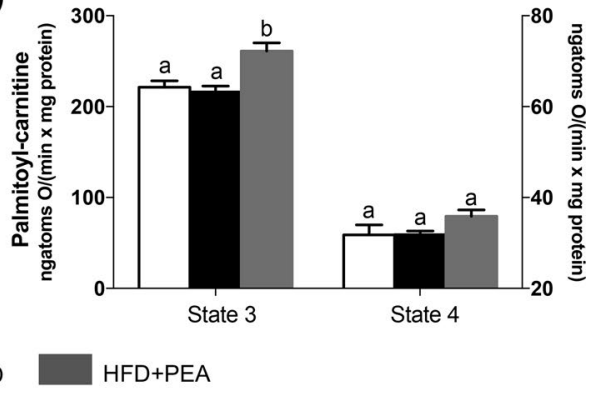

(D)

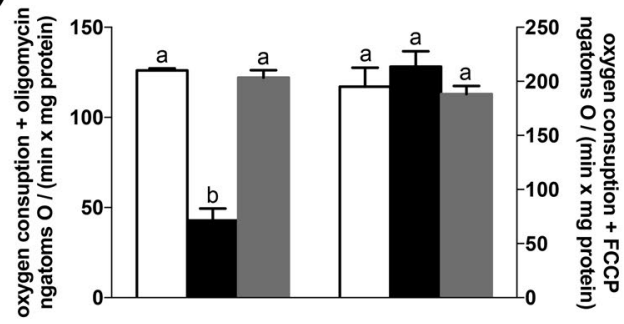

(F)

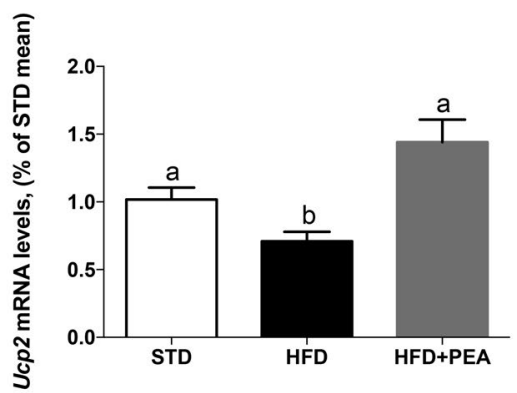

F I G U RE 5 Effect of PEA on liver mitochondrial function and energy efficiency. A, Mitochondrial respiration in the presence of succinate or B, Palmitoyl-carnitine, as substrates, was determined in presence of ADP (state 3) and in the presence of substrates alone (state 4). C, CPT activity was determined in isolated liver mitochondria. The oxygen consumption rate was evaluated $\mathrm{D}$, In the presence of oligomycin, that inhibits ATP synthase, and FCCP, which dissipates trans-mitochondrial proton gradient. E, The degree of coupling is also shown. F, mRNA levels of UCP2 were also reported. Data are presented as means \pm SEM of animals from different groups $(\mathrm{n}=4-6$ each group). Labeled means without a common letter differ, $P<.05$ 


\section{5 | PEA counteracts mitochondrial dysfunction in palmitate-challenged HepG2 cells}

PEA effect on mitochondrial bioenergetics in Pal-treated cells was assessed using Mito Stress assay by Seahorse analyzer (Figure 7A). PEA treatment recovered Pal-induced mitochondrial dysfunction, increasing basal and maximal respiration (Figure 7B,C), promoting ATP-linked respiration and proton leak (Figure 7D,E), and reducing mitochondrial coupling efficiency (Figure 7F). All these data indicate the direct effect of PEA in counteracting Pal-induced mitochondrial dysfunction.

\section{6 | Involvement of AMPK in PEA effect on lipid metabolism in HepG2 cells}

The direct lowering effect of PEA on hepatic lipid accumulation induced by Pal was shown on HepG2 cells (Figure 8A).

(A)
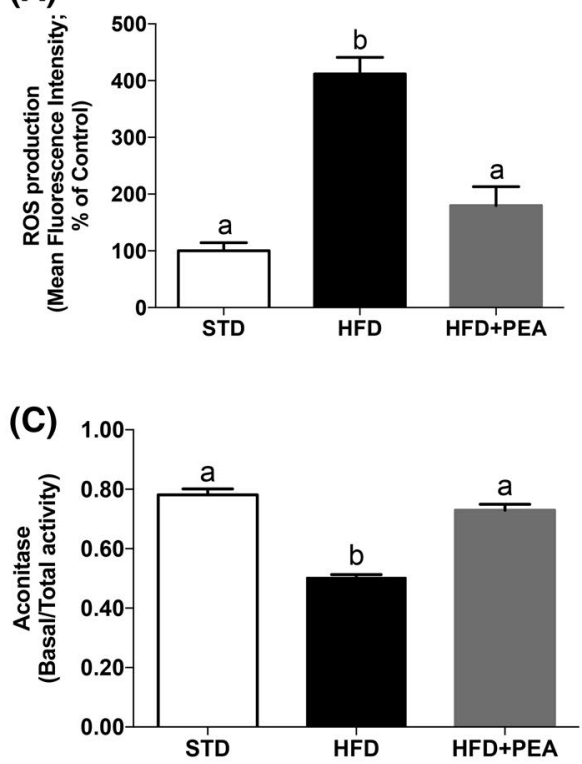

(E)

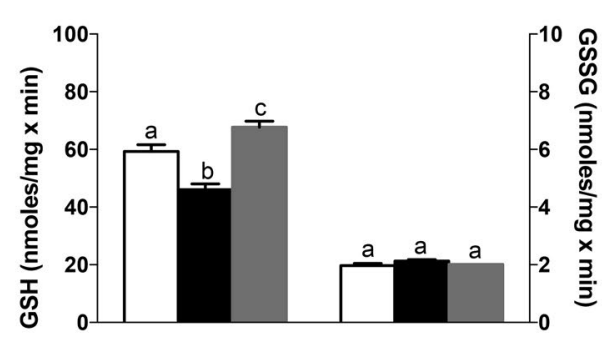

Notably, the PEA effect was blunted by compound C, a potent inhibitor of AMPK (Figure 8A). Indeed, at the mechanistic level, PEA restored AMPK phosphorylation and increased PPAR- $\alpha$ and CPT1 expression in Pal-challenged HepG2 (Figure 8B). All these effects were reversed in the presence of compound $\mathrm{C}$ (Figure 8C), clarifying the key role of AMPK in PEA metabolic activity.

\section{4 | DISCUSSION}

This study established the modulatory effect of PEA on metabolic inflexibility induced in HFD-fed mice, showing PEA capability to restore glucose and lipid homeostasis. This lipid mediator belongs to bioactive $\mathrm{N}$-acylethanolamines (NAEs) and is involved in many physiopathological processes, revealing pleiotropic activities and carrying out anti-inflammatory, analgesic and neuroprotective effects. ${ }^{36,37}$ Differently from the most known NAE anandamide, that binds cannabinoid receptor, PEA effects are mainly due to its interaction with

(B)
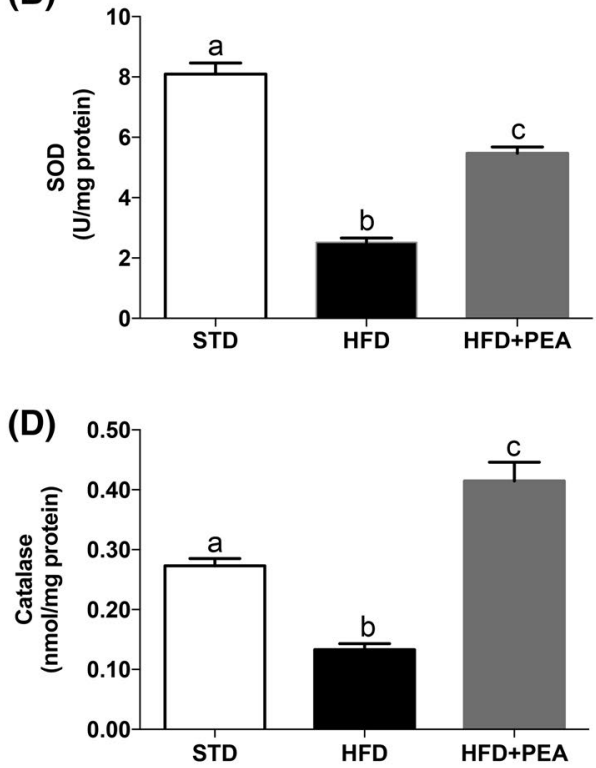

(F)

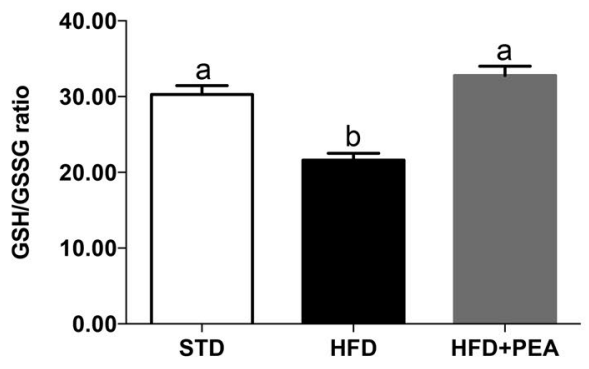

F I G U R E 6 PEA improves antioxidant/detoxifying defense in liver of HFD-fed mice. A, ROS production, B, SOD, C, Aconitase, D, Catalase activity is reported. E, GSH and GSSG content, and F, The GSH-to-GSSG ratio are shown. Data are presented as means \pm SEM of all animals from different groups ( $\mathrm{n}=6$ each group). Labeled means without a common letter differ, $P<.05$ 
PPAR- $\alpha .{ }^{38,39}$ The present study identifies a novel function for PEA, as an insulin sensitizer based on its effects in regulating glucose homeostasis, and managing obesity, liver steatosis, and IR.

We have demonstrated that PEA limits overweight and adiposity induced by HFD, reducing energy intake, increasing energy expenditure, resting metabolic rate, and lipid oxidation, as shown by the reduced RQ. Indeed, the decreased RQ index reflects the increased use of fatty acids, rather than carbohydrate, as the primary fuel, dissipating most part of energy gained from HFD. In obese mice, PEA-induced shift toward lipid oxidation determines an improvement of lipid and inflammatory profile, limiting the progression of hepatic steatosis.

PEA treatment also impacts on glucose homeostasis and IR. Indeed, our data show an improved capacity in glucose disposal and a decrease in HOMA-IR. To assess the intracellular mechanisms, we studied the InsR-AKT pathway in the liver. The insulin-sensitizing effect of PEA is demonstrated by the increase in the phosphorylated state of InsR and AKT, resulting in upregulation of GLUT2 and subsequent glucose clearance.

Recently, the activation of AMPK, a cellular energy sensor, has been identified as a therapeutic target for treating metabolic diseases. ${ }^{40}$ It leads to the phosphorylation of key metabolic mediators and transcriptional regulators that are linked to cellular metabolism including PPARs. ${ }^{41}$ Indeed, AMPK activation reprograms cells, redirecting metabolism toward inhibited anabolism and increased catabolism, limiting glucose and lipid synthesis and promoting FAO as an energy source. In our experimental conditions, PEA, increasing liver AMPK phosphorylation, reduced lipid synthesis, not only by the inhibitory phosphorylation of ACC but also decreasing fatty acid synthase transcription and inducing PPAR- $\alpha$ expression. Notably, PEA induced PPAR- $\alpha$ both in vivo and in vitro model, increasing also its transcriptional coactivator PGC1 $\alpha$. Consistently, our previous data evidenced PEA capability to increase AMPK activation in adipose tissue, to inhibit cytokine synthesis, and to reduce the activated state of macrophages. Indeed, PEA modulated AMPK phosphorylation both in hypothalamus and white adipose tissue of ovariectomized rats, leading to a reduction of food intake and fat mass. ${ }^{16}$ Previous data demonstrated that activation of AMPK can inhibit the synthesis of proinflammatory cytokines (ie, TNF- $\alpha$ and IL-6) both in macrophages ${ }^{42,43}$ and in adipocytes. $^{44}$

Here, in HFD mice, PEA improves alteration of serum leptin and adiponectin, deeply reducing their ratio. Interestingly, both adipokines inversely modulate glucose and lipid metabolism through AMPK signaling. ${ }^{45,46}$ Metabolic diseases and related comorbidities result from the overproduction of lipids derived by de novo lipogenesis and from the inability to oxidize lipids stored in the liver. ${ }^{40}$ These effects are counteracted by AMPK/
ACC pathway activation: in fact, the overexpression of AMPK reduces lipogenic gene expression, liver triglycerides, and hepatic steatosis in hyperlipidemic diabetic rats ${ }^{47}$ and reduces intracellular lipid accumulation in hepatocyte cells. ${ }^{48}$

As known, AMPK inhibits fatty acid synthesis by inducing the inhibitory phosphorylation of two targets: ACC1, which catalyzes the rate-limiting step in fatty acid synthesis converting acetyl-CoA to malonyl-CoA, and sterol regulatory element-binding protein $1 \mathrm{c}$, a transcription factor that induces the expression of multiple lipogenic enzymes, including ACC1 and fatty acid synthase. ${ }^{49}$ Besides the inhibition of lipid anabolism, AMPK also activates lipid catabolism. The AMPK-induced phosphorylation of ACC determines the reduction of malonyl CoA, which, in turn, disinhibits CPT activity, resulting in increased mitochondrial FAO. ${ }^{50}$ ACC knock-in mice fed a control diet rapidly develop NAFLD, IR, and liver fibrosis ${ }^{51}$; conversely, the pharmacological inhibition of ACC reduces lipogenesis, increases FAO in isolated hepatocytes, and alleviates NAFLD in rats. ${ }^{52}$

As previously demonstrated, HFD feeding, inducing hepatic fat storage, dysregulated mitochondrial function. ${ }^{53}$ Accordingly, our data demonstrate that liver mitochondria from the HFD mice exhibited reduced mitochondrial respiratory capacity and increased oxidative stress. PEA treatment improves mitochondrial respiratory capacity and FAO and stimulated CPT activity. These effects lead to the reduction in hepatic ectopic lipid storage, as shown by triglycerides content and Oil Red O staining. Moreover, a further mechanism converging to fatty oxidation is related to the decrease in mitochondrial efficiency, as shown by the decrease in the degree of coupling in PEA-treated HFD mice. The reduced degree of coupling associated to increased transcription of UCP2 contributed to the decrease in oxidative stress (ie, reduction of ROS production, increase in aconitase activity and GSH/GSSG ratio) and an increase of detoxifying enzymes (ie, catalase and SOD activity) in PEA-treated HFD mice.

In vitro data on the effect of PEA on mitochondrial function obtained in palmitate-challenged HepG2 cells showed similar results, since we observed an increase in basal and maximal respiration and in ATP-linked respiration. Moreover, PEA exposure induced an increase in proton leak, and subsequently a reduction of coupling efficiency.

In our in vitro experiments, we also addressed the direct effect of PEA on hepatic lipid metabolism through AMPKdependent mechanism using compound $\mathrm{C}$, a potent and selective inhibitor of the enzyme. As, known, HepG2 cells treated with palmitate have been commonly used to study the impairment of hepatic lipid metabolism and steatosis related to NAFLD $^{54-56}$ and represent an appropriate tool to determine the involvement of AMPK activation in PEA effects. PEA treatment reduced lipid accumulation induced by palmitate in HepG2 cells, activating the phosphorylation of AMPK, and the expression of PPAR- $\alpha$ and CPT1. Notably, PEA effects 
(A)

Cell Mito Stress Test Profile

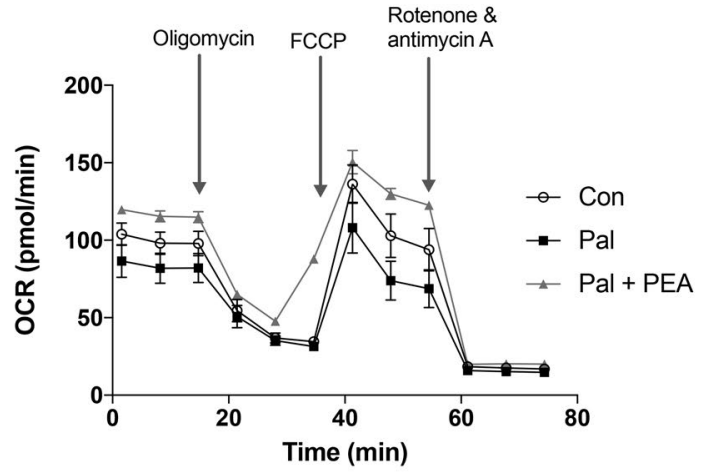

(C)

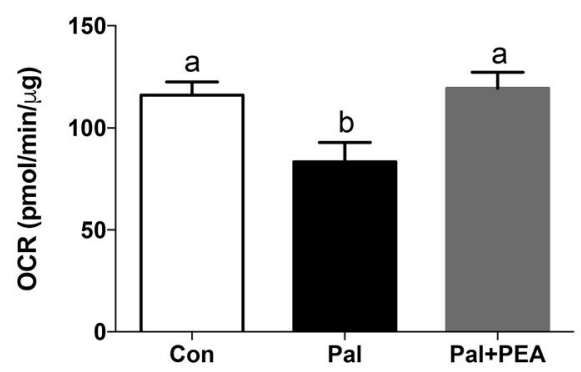

(E)

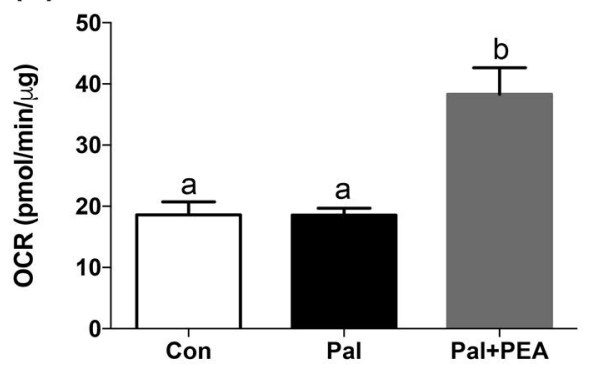

(B)

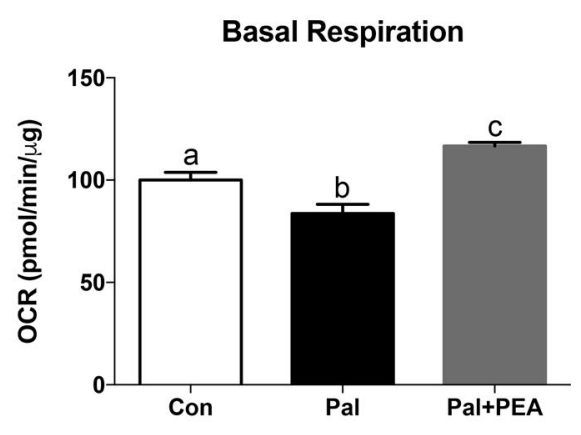

(D)

ATP-linked Respiration

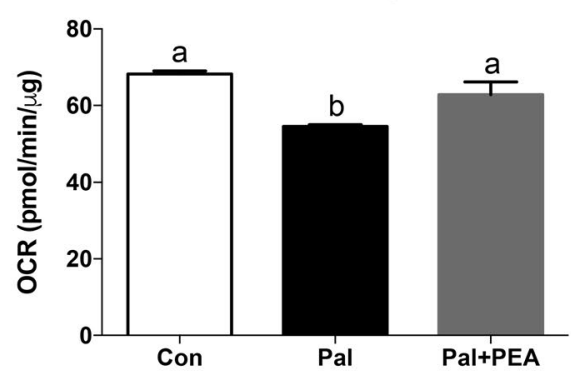

(F)

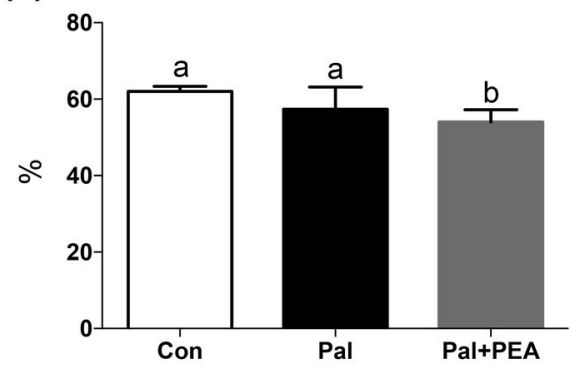

F I G U RE 7 PEA effect on mitochondrial function of Pal-challenged HepG2 cells. A, Basal oxygen consumption rate (OCR) was determined by Cell Mito Stress Test in Sea Horse analyzer, following the addition of oligomycin, the uncoupler FCCP, rotenone and antimycin A, inhibitors of complexes I and III, respectively. B, Basal respiration, C, maximal respiration, D, ATP-linked respiration, E, proton leak, as well as F, coupling efficiency were calculated in HepG2 cells stimulated with Pal $(100 \mu \mathrm{M})$ in presence or not of PEA $(1 \mu \mathrm{M})$. OCR was normalized to the protein content of each well for all measurements by Bradford assay. Data are the mean \pm SEM of three different experiments with three technical replicates. Labeled means without a common letter differ, $P<.05$

were blunted by AMPK inhibition, evidencing the involvement of this enzyme in PEA-induced improvement of lipid metabolism altered by palmitate.

As known, PPAR- $\alpha$ is an intriguing therapeutic target for fatty liver disease but novel efficient and safe agents stimulating PPAR- $\alpha$ are needed; indeed, the fibrates, regarded available PPAR- $\alpha$ activators, have not reported beneficial clinical effect in the treatment of NAFLD/NASH ${ }^{57}$ Here, we showed that oral administration of PEA induced not only an increase in PEA level but also PPAR- $\alpha$ expression in fatty liver indicating the direct activation of PEA/PPAR- $\alpha$ pathway. Interestingly, it has been already described that PEA levels decreased in rat fatty liver due to overfeeding ${ }^{58}$ as well as PEA plasma levels in insulin-resistant obese women ${ }^{59}$; it is likely that PEA levels, due to its biosynthesis/degradation, are modulated by insulin sensitivity.

It has been demonstrated that ultramicronized PEA formulation is promptly absorbed. ${ }^{60}$ Even if this exogenous administration increases PEA levels in liver of HFD mice, we did not detect any change either of palmitic or palmitoleic or oleic acid content. In addition, it should be considered that the amount of palmitic acid derived by PEA degradation is negligible respect to whole tissue concentration, which is tightly controlled around a well-defined concentration and depends on exogenous source and endogenous biosynthesis. ${ }^{61}$

Based on our results, PEA can be considered a therapeutic tool to improve metabolic flexibility impaired by 
(A)

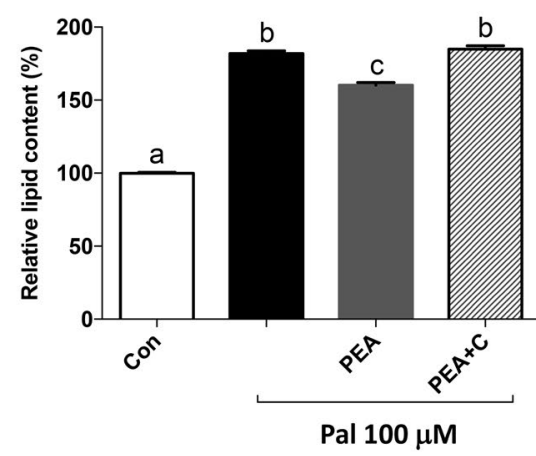

(B)

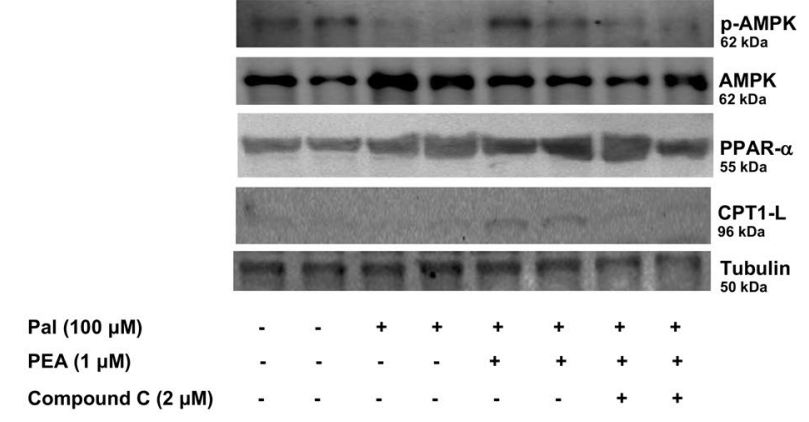

(C)
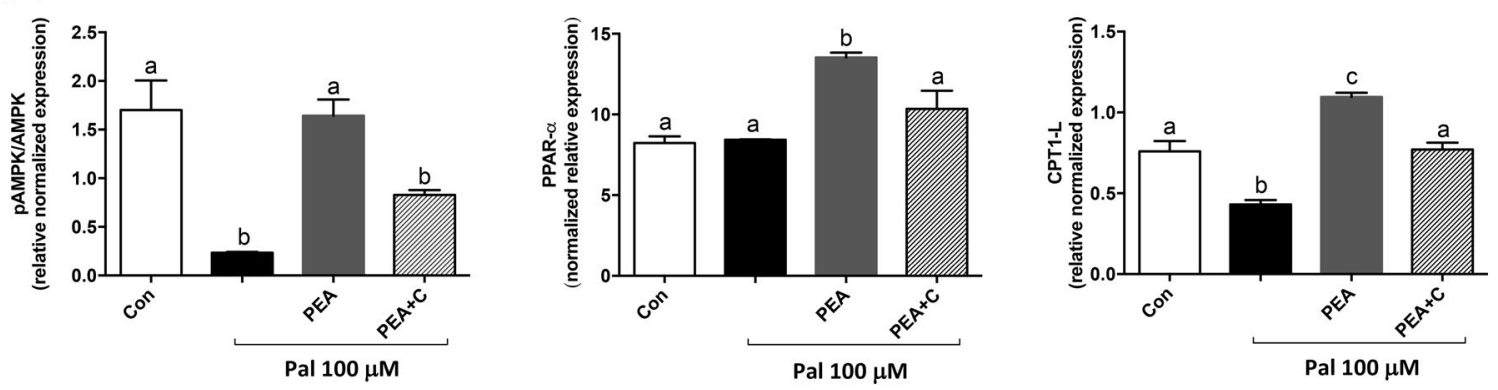

F I G U RE 8 Involvement of AMPK in PEA effect on lipid metabolism in HepG2 cells. A, Lipid content was assessed in Pal-challenged HepG2 cells, treated or not with PEA or compound C, an inhibitor of AMPK. B, Protein expression of pAMPK-to-AMPK, PPAR- $\alpha$, CPT1 was reported. C, Densitometric analysis of pAMPK-to-AMPK, PPAR- $\alpha$, CPT1 was also determined. Data are from three different experiments in duplicate and presented as means \pm SEM. Labeled means without a common letter differ, $P<.05$

obesity. The involvement of AMPK in metabolic effects of PEA identifies this molecule as lipid and glucose metabolic regulator, relevant in the modulation of liver insulin sensitivity. The beneficial effects of PEA may be the result of multiple converging mechanisms: the improvement of mitochondrial respiratory capacity and FAO, the decrease in mitochondrial efficiency, the improvement of liver redox status associated to cytoprotective defenses and its wellknown anti-inflammatory effect.

\section{ACKNOWLEDGMENTS}

We thank Mr. Giovanni Esposito, Mr. Angelo Russo, and Dr. Antonio Baiano for animal care and technical assistance.

\section{AUTHOR CONTRIBUTIONS}

R. Meli, G. Mattace Raso, and M.P. Mollica designed the research; C. Annunziata, A. Lama, C. Pirozzi, G. Cavaliere, G. Trinchese, F. Di Guida, A. Nitrato Izzo, F. Cimmino, O. Paciello, D. De Biase, and E. Murru performed experiments; C. Annunziata, A. Lama, C. Pirozzi, G. Cavaliere, G. Trinchese, O. Paciello, and S. Banni analyzed data; R. Meli, G. Mattace Raso, and M.P. Mollica wrote the paper; G. Mattace Raso, M.P. Mollica, and A. Calignano supervised the study.

\section{CONFLICT OF INTEREST}

The authors declare that they have no conflict of interest.

\section{REFERENCES}

1. Smith RL, Soeters MR, Wust RCI, Houtkooper RH. Metabolic flexibility as an adaptation to energy resources and requirements in health and disease. Endocr Rev. 2018;39:489-517.

2. Battaglia GM, Zheng D, Hickner RC, Houmard JA. Effect of exercise training on metabolic flexibility in response to a highfat diet in obese individuals. Am J Physiol Endocrinol Metab. 2012;303:E1440-E1445.

3. Morino K, Petersen KF, Shulman GI. Molecular mechanisms of insulin resistance in humans and their potential links with mitochondrial dysfunction. Diabetes. 2006;55(Suppl 2):S9-S15.

4. Theurey P, Rieusset J. Mitochondria-associated membranes response to nutrient availability and role in metabolic diseases. Trends Endocrinol Metab. 2017;28:32-45.

5. Begriche K, Massart J, Robin MA, Bonnet F, Fromenty B. Mitochondrial adaptations and dysfunctions in nonalcoholic fatty liver disease. Hepatology. 2013;58:1497-1507.

6. Khan R, Bril F, Cusi K, Newsome PN. Modulation of insulin resistance in NAFLD. Hepatology. 2019;70:711-724.

7. Obre E, Rossignol R. Emerging concepts in bioenergetics and cancer research: metabolic flexibility, coupling, symbiosis, switch, oxidative tumors, metabolic remodeling, signaling and bioenergetic therapy. Int J Biochem Cell Biol. 2015;59:167-181. 
8. Meli R, Mattace Raso G, Calignano A. Role of innate immune response in non-alcoholic fatty liver disease: metabolic complications and therapeutic tools. Front Immunol. 2014;5:177.

9. Konrad D, Wueest S. The gut-adipose-liver axis in the metabolic syndrome. Physiology (Bethesda). 2014;29:304-313.

10. Bensinger SJ, Tontonoz P. Integration of metabolism and inflammation by lipid-activated nuclear receptors. Nature. 2008; 454:470-477.

11. D'Agostino G, Cristiano C, Lyons DJ, et al. Peroxisome proliferator-activated receptor alpha plays a crucial role in behavioral repetition and cognitive flexibility in mice. Mol Metab. 2015;4:528-536.

12. Gross B, Pawlak M, Lefebvre P, Staels B. PPARs in obesityinduced T2DM, dyslipidaemia and NAFLD. Nat Rev Endocrinol. 2017;13:36-49.

13. Franko A, Huypens $P$, Neschen S, et al. Bezafibrate improves insulin sensitivity and metabolic flexibility in STZ-induced diabetic mice. Diabetes. 2016;65:2540-2552.

14. Wallenius K, Kjellstedt A, Thalen P, Lofgren L, Oakes ND. The PPAR alpha/gamma agonist, tesaglitazar, improves insulin mediated switching of tissue glucose and free fatty acid utilization in vivo in the obese zucker rat. PPAR Res. 2013;2013:305347.

15. Petrosino S, Di Marzo V. The pharmacology of palmitoylethanolamide and first data on the therapeutic efficacy of some of its new formulations. Br J Pharmacol. 2017;174:1349-1365.

16. Mattace Raso G, Santoro A, Russo R, et al. Palmitoylethanolamide prevents metabolic alterations and restores leptin sensitivity in ovariectomized rats. Endocrinology. 2014;155:1291-1301.

17. Fanelli F, Mezzullo M, Repaci A, et al. Profiling plasma $\mathrm{N}$-acylethanolamine levels and their ratios as a biomarker of obesity and dysmetabolism. Mol Metab. 2018;14:82-94.

18. Dominguez JF, Guo L, Carrasco Molnar MA, et al. Novel indirect calorimetry technology to analyze metabolism in individual neonatal rodent pups. PLoS ONE. 2009;4:e6790.

19. Piscitelli F, Carta G, Bisogno T, et al. Effect of dietary krill oil supplementation on the endocannabinoidome of metabolically relevant tissues from high-fat-fed mice. Nutr Metab (Lond). 2011;8:51.

20. Lama A, Pirozzi C, Mollica MP, et al. Polyphenol-rich virgin olive oil reduces insulin resistance and liver inflammation and improves mitochondrial dysfunction in high-fat diet fed rats. Mol Nutr Food Res. 2017;61:1600418.

21. Cairns CB, Walther J, Harken AH, Banerjee A. Mitochondrial oxidative phosphorylation thermodynamic efficiencies reflect physiological organ roles. Am J Physiol. 1998;274:R1376-R1383.

22. Cavaliere G, Trinchese G, Bergamo P, et al. Polyunsaturated fatty acids attenuate diet induced obesity and insulin resistance, modulating mitochondrial respiratory uncoupling in rat skeletal muscle. PLoS One. 2016;11:e0149033.

23. Alexson SE, Nedergaard J. A novel type of short- and medium-chain acyl-CoA hydrolases in brown adipose tissue mitochondria. J Biol Chem. 1988;263:13564-13571.

24. Flohe L, Otting F. Superoxide dismutase assays. Methods Enzymol. 1984;105:93-104.

25. Aebi H. Catalase in vitro. Methods Enzymol. 1984;105:121-126.

26. Bergamo P, Maurano F, Rossi M. Phase 2 enzyme induction by conjugated linoleic acid improves lupus-associated oxidative stress. Free Radic Biol Med. 2007;43:71-79.

27. Cao J, Dai DL, Yao L, et al. Saturated fatty acid induction of endoplasmic reticulum stress and apoptosis in human liver cells via the PERK/ATF4/CHOP signaling pathway. Mol Cell Biochem. 2012;364:115-129.
28. Wu D, Liu J, Pang X, et al. Palmitic acid exerts pro-inflammatory effects on vascular smooth muscle cells by inducing the expression of C-reactive protein, inducible nitric oxide synthase and tumor necrosis factor-alpha. Int J Mol Med. 2014;34: 1706-1712.

29. Ferrante MC, Amero P, Santoro A, et al. Polychlorinated biphenyls (PCB 101, PCB 153 and PCB 180) alter leptin signaling and lipid metabolism in differentiated 3T3-L1 adipocytes. Toxicol Appl Pharmacol. 2014;279:401-408.

30. Brand MD, Nicholls DG. Assessing mitochondrial dysfunction in cells. Biochem J. 2011;435:297-312.

31. Divakaruni AS, Paradyse A, Ferrick DA, Murphy AN, Jastroch M. Analysis and interpretation of microplate-based oxygen consumption and pH data. Methods Enzymol. 2014;547:309-354.

32. Kleiner DE, Brunt EM, Van Natta M, et al. Design and validation of a histological scoring system for nonalcoholic fatty liver disease. Hepatology. 2005;41:1313-1321.

33. Oriente F, Cabaro S, Liotti A, et al. PREP1 deficiency downregulates hepatic lipogenesis and attenuates steatohepatitis in mice. Diabetologia. 2013;56:2713-2722.

34. Lama A, Annunziata C, Coretti L, et al. N-(1-carbamoyl-2phenylethyl) butyramide reduces antibiotic-induced intestinal injury, innate immune activation and modulates microbiota composition. Sci Rep. 2019;9:4832.

35. Mailloux RJ, Harper ME. Uncoupling proteins and the control of mitochondrial reactive oxygen species production. Free Radic Biol Med. 2011;51:1106-1115.

36. Alhouayek M, Muccioli GG. Harnessing the anti-inflammatory potential of palmitoylethanolamide. Drug Discov Today. 2014;19:1632-1639.

37. Russo R, Cristiano C, Avagliano C, et al. Gut-brain axis: role of lipids in the regulation of inflammation, pain and CNS diseases. Curr Med Chem. 2018;25:3930-3952.

38. Mattace Raso G, Russo R, Calignano A, Meli R. Palmitoylethanolamide in CNS health and disease. Pharmacol Res. 2014;86:32-41.

39. Lo Verme J, Fu J, Astarita G, et al. The nuclear receptor peroxisome proliferator-activated receptor-alpha mediates the anti-inflammatory actions of palmitoylethanolamide. Mol Pharmacol. 2005;67:15-19.

40. Day EA, Ford RJ, Steinberg GR. AMPK as a therapeutic target for treating metabolic diseases. Trends Endocrinol Metab. 2017;28:545-560.

41. Herzig S, Shaw RJ. AMPK: guardian of metabolism and mitochondrial homeostasis. Nat Rev Mol Cell Biol. 2018;19:121-135.

42. Yang Z, Kahn BB, Shi H, Xue BZ. Macrophage alpha1 AMPactivated protein kinase (alpha1AMPK) antagonizes fatty acid-induced inflammation through SIRT1. J Biol Chem. 2010;285:19051-19059.

43. Sag D, Carling D, Stout RD, Suttles J. Adenosine 5'-monophosphate-activated protein kinase promotes macrophage polarization to an anti-inflammatory functional phenotype. J Immunol. 2008; 181:8633-8641.

44. Lihn AS, Pedersen SB, Lund S, Richelsen B. The anti-diabetic AMPK activator AICAR reduces IL-6 and IL-8 in human adipose tissue and skeletal muscle cells. Mol Cell Endocrinol. 2008;292:36-41.

45. Minokoshi Y, Kim YB, Peroni OD, et al. Leptin stimulates fatty-acid oxidation by activating AMP-activated protein kinase. Nature. 2002;415:339-343. 
46. Long YC, Zierath JR. AMP-activated protein kinase signaling in metabolic regulation. J Clin Invest. 2006;116:1776-1783.

47. Seo E, Park EJ, Joe Y, et al. Overexpression of AMPKalpha1 ameliorates fatty liver in hyperlipidemic diabetic rats. Korean J Physiol Pharmacol. 2009;13:449-454.

48. Zhang HA, Yang XY, Xiao YF. AMPKalpha1 overexpression alleviates the hepatocyte model of nonalcoholic fatty liver disease via inactivating p38MAPK pathway. Biochem Biophys Res Commun. 2016;474:364-370.

49. Jeon SM. Regulation and function of AMPK in physiology and diseases. Exp Mol Med. 2016;48:e245.

50. Kahn BB, Alquier T, Carling D, Hardie DG. AMP-activated protein kinase: ancient energy gauge provides clues to modern understanding of metabolism. Cell Metab. 2005;1:15-25.

51. Fullerton MD, Galic S, Marcinko K, et al. Single phosphorylation sites in Acc1 and Acc2 regulate lipid homeostasis and the insulin-sensitizing effects of metformin. Nat Med. 2013;19:1649-1654

52. Harriman G, Greenwood J, Bhat S, et al. Acetyl-CoA carboxylase inhibition by ND-630 reduces hepatic steatosis, improves insulin sensitivity, and modulates dyslipidemia in rats. Proc Natl Acad Sci USA. 2016;113:E1796-1805.

53. Mollica MP, Mattace Raso G, Cavaliere G, et al. Butyrate regulates liver mitochondrial function, efficiency, and dynamics in insulin-resistant obese mice. Diabetes. 2017;66:1405-1418.

54. Gomez-Lechon MJ, Donato MT, Martinez-Romero A, Jimenez N, Castell JV, O'Connor JE. A human hepatocellular in vitro model to investigate steatosis. Chem Biol Interact. 2007;165:106-116.

55. Kanuri G, Bergheim I. In vitro and in vivo models of non-alcoholic fatty liver disease (NAFLD). Int J Mol Sci. 2013;14:11963-11980.

56. Kim K, Bae GD, Lee M, et al. Allomyrina dichotoma larva extract ameliorates the hepatic insulin resistance of high-fat diet-induced diabetic mice. Nutrients. 2019;11(7):1522.
57. Tanaka N, Aoyama T, Kimura S, Gonzalez FJ. Targeting nuclear receptors for the treatment of fatty liver disease. Pharmacol Ther. 2017; 179:142-157.

58. Izzo AA, Piscitelli F, Capasso R, et al. Basal and fasting/refeeding-regulated tissue levels of endogenous PPAR-alpha ligands in Zucker rats. Obesity (Silver Spring). 2010;18:55-62.

59. Abdulnour J, Yasari S, Rabasa-Lhoret R, et al. Circulating endocannabinoids in insulin sensitive vs. insulin resistant obese postmenopausal women. A MONET group study. Obesity. 2014;22: 211-216.

60. Petrosino S, Cordaro M, Verde R, et al. Oral ultramicronized palmitoylethanolamide: plasma and tissue levels and spinal antihyperalgesic effect. Front Pharmacol. 2018;9:249.

61. Carta G, Murru E, Banni S, Manca C. Palmitic acid: physiological role, metabolism and nutritional implications. Front Physiol. 2017;8:902.

\section{SUPPORTING INFORMATION}

Additional supporting information may be found online in the Supporting Information section.

How to cite this article: Annunziata C, Lama A, Pirozzi C, et al. Palmitoylethanolamide counteracts hepatic metabolic inflexibility modulating mitochondrial function and efficiency in diet-induced obese mice. The FASEB Journal. 2020;34:350-364. https://doi.org/10.1096/fj.201901510RR 